\title{
Perspektiven und Entwicklungen in der Radioonkologie der letzten 25 Jahre
}

\author{
T.G. Wendt \\ Radiologische Klink und Poliklinik, Friedrich-Schiller-Universität Jena
}

Das zurückliegende Vierteljahrhundert - die Zeitspanne des Bestehens von ONKOLOGIE - ist geprägt von einem bemerkenswerten Wandel der Radioonkologie in vielfacher Hinsicht. Maßgebliche Änderungen in den Bereichen

- Technologie der Bestrahlungsanlagen,

- moderne Methoden der Schnittbildverfahren als Basis der physikalisch-anatomischen Bestrahlungsplanung,

- Verfügbarkeit leistungsfähiger Computertechnologie,

- radiobiologische Erkenntnisse an Tumor- und Normalgeweben,

- Interaktion und klinischer Einsatz von ionisierenden Strahlen und dosismodifizierenden Substanzen (Zytostatika, Radiosensitizer),

- Integration der Radioonkologie in multimodale Therapiekonzepte und

- Funktions- und Organerhalt durch kurative Strahlentherapie

bewirkten eine dramatische Entwicklung des Fachgebiets. Der Neologismus «Radioonkologie» bringt zudem das gewachsene Selbstverständnis im Kanon der onkologischen Disziplinen zum Ausdruck. Er rückt die klinische Orientierung des Fachgebiets gegenüber den methodisch-physikalischen Aspekten in den Vordergrund.

\section{Wandel der Technologien der Bestrahlungsanlagen}

Nachdem 1956 das erste Betatron in Göttingen und 1959 in München die erste Telekobalt-Anlage installiert worden waren, standen Mitte der 1970er Jahre die ersten Linearbeschleuniger in Deutschland für die klinische Nutzung zur Verfügung. Ihre Vorteile liegen in der hohen Dosisleistung, was gegenüber dem Kreisbeschleuniger die Bestrahlungsdauer für ein Feld von 4-8 min auf unter 1 min verkürzt, sowie in der größeren Eindringtiefe der hochenergetischen Photonen ge-

\begin{tabular}{ll}
\hline KARGER & ( ) 2002 S. Karger GmbH, Freiburg \\
Fax +497614520714 & Accessible online at: \\
$\begin{array}{l}\text { E-mail Information@Karger.de } \\
\text { www.karger.com }\end{array}$ & www.karger.com/journals/onk
\end{tabular}

genüber Telekobalt-Gammastrahlen. Bewegliche Feldblenden erlauben eine weitaus bessere Anpassung der bestrahlten Volumina an die Zielvolumenausdehnung. Die in den 1990er Jahren begonnene Entwicklung und klinische Implementierung von Lamellenkollimatoren führt zu einer weiteren Verminderung der unerwünschten Mitbestrahlung von Normalgeweben, was zu einer Verminderung von akuten und chronischen Therapiefolgen führen wird. Die miniaturisierte Ausführung (Mikro-«multi-leaf»-Kollimator) beschleunigt den Ablauf der stereotaktischen Radiochirurgie (Einzeitbestrahlung mit hohen Dosen) wie auch der stereotaktischen Radiotherapie (konventionell fraktionierte Strahlentherapie). Wenngleich die Nachweise darüber im Sinne evidenzbasierter Medizin als unethisch einzustufen sein dürften, müssen heute an eine kurativ intendierte, hochdosierte (sogenannte radikale) Strahlentherapie hohe apparative Forderungen gestellt werden. Erst diese neue Technologie der Feldkollimation erlaubt die intensitätsmodulierte Radiotherapie (IMRT) in «Step and shoot»-Technik sowie mit dynamischer Technik («sliding window»), die seit wenigen Jahren in klinischer Erprobung ist und einen Meilenstein in der technologischen Entwicklung darstellt.

\section{Inkorporation moderner Schnittbildverfahren in die} physikalisch-anatomische Bestrahlungsplanung

Rasch nach Installation der ersten Computertomographen Mitte der 1970er Jahre lösten Computertomographie(CT)-basierte Rechenprogramme zur Bestimmung der Dosisverteilung im durchstrahlten Gewebe die bis dahin übliche Tiefendosiskalkulation ab. Die CT erlaubt die volle Berücksichtigung nicht nur der anatomischen Binnenstrukturen, sondern auch ihrer spezifischen Dosisabsorptionseigenschaften und ist die Voraussetzung für die heute routinemäßig durchgeführte 

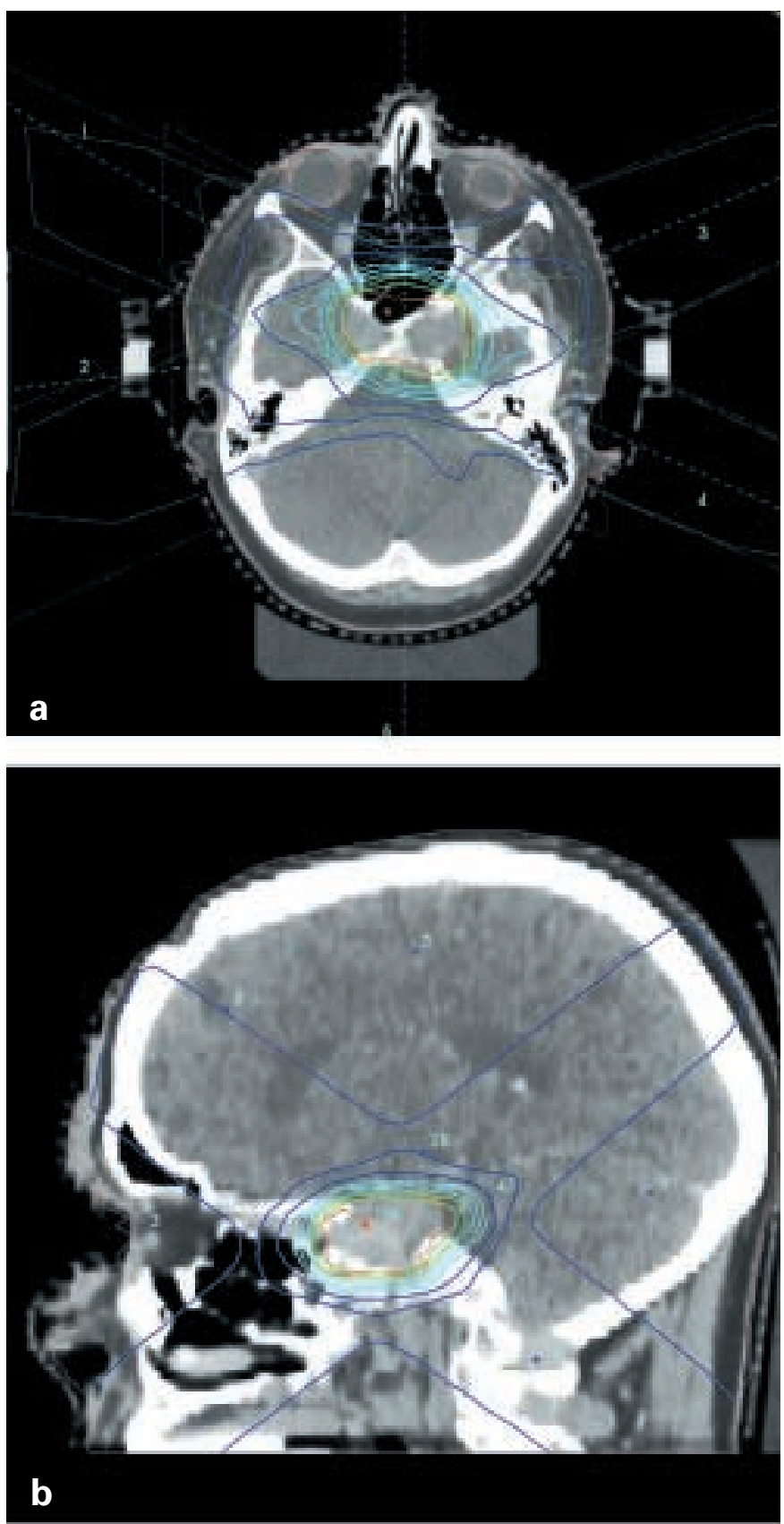

Abb. 1. Dosisverteilung (grüne Isodosenlinien: $95 \%$ und $100 \%$ ) bei nonkoplanarer Bestrahlung eines Hypophysentumors. Steiler Dosisgradient vom Zielvolumenrand zum umgebenden gesunden Hirngewebe. a Transversale Rekonstruktion, b sagittale Rekonstruktion.

quantitative Abschätzung von zu erwartenden chronischen Normalgewebsreaktionen, was sicher maßgeblich zur Verminderung der Spättherapiefolgen bei geheilten Patienten beiträgt. Um die spezifischen Informationen aus metabolischen Untersuchungen wie der Positronenemissionstomographie (PET) und der Single-Photon-Emissionscomputertomographie (SPECT), aber auch Informationen aus der Kernspintomographie zu nutzen, werden bei speziellen Fragestellungen
Bildinformationen mit der CT elektronisch übereinandergelagert [1]. Insbesondere bei der Strahlentherapie von benignen und semimalignen Tumoren im Bereich des Kraniums ist das «Matching» von CT und Magnetresonanztomographie (magnetic resonance imaging; MRI) heute unabdingbar. Erst der Einsatz leistungsfähiger Computer erlaubt die Isodosendarstellung und -optimierung in allen beliebigen Schnittebenen, was insbesondere bei der Planung der Radiochirurgie essenziell ist.

\section{Verfügbarkeit leistungsfähiger Computertechnologie}

Die Verfügbarkeit von zunehmend leistungsfähigeren Computern trug und trägt in vielen Teilbereichen zu einer Qualitätsverbesserung bei. Sie ermöglichte ab Ende der 1980er Jahre die dreidimensionale Bestrahlungsplanung und deren Optimierung anhand der quantitativen Analyse von DosisVolumen-Histogrammen. Durch die Möglichkeit der Planung und Dosisberechnung von nichtkoplanaren Feldern (d.h. aus beliebigen Raumwinkeln, drei oder mehr Felder liegen nicht in einer Ebene) wurde die Anpassung des bestrahlten Volumens an das Zielvolumen vielfach verbessert (Abb. 1). Erst die computerisierte Steuerung des Bestrahlungsablaufs erlaubt die IMRT. Bei der «Step and shoot»-Methode werden 40-80 Lamellen eines den Photonenstrahl eingrenzenden Kollimators so gesteuert, dass die Dosisverteilung innerhalb eines Bestrahlungsfelds in weiten Grenzen variiert werden kann (Abb. 2) - mit dem Ziel bewusster Dosismodifikation in Zielvolumen. Bei der in Entwicklung begriffenen IMRT durch 3-D-Kompensator wird im Planungsrechner für jedes einzelne Bestrahlungsfeld ein Fluenzprofil vorausberechnet. Anschließend wird entsprechend dieses Profils ein 3-D-Metallkompensator hergestellt, der bei jeder Bestrahlung die Dosisverteilung spezifisch modifiziert [2] (Abb. 3). In der Summe resultiert eine Schonung von Normalgeweben (Abb. 4).

\section{Radiobiologische Erkenntnisse an Tumor- und Normalgeweben}

Zu Beginn der 1970er Jahre war die klinische Strahlentherapie mit 5 Fraktionen pro Woche allgemein geübte Praxis. Mitte der 1980er Jahre wurden aus tumor- und strahlenbiologischen Überlegungen heraus andere (akzelerierte, hyperfraktionierte Bestrahlung, «Concomitant boost»-Konzept) Fraktionierungsschemata eingesetzt, die teilweise $\mathrm{zu}$ besseren Tumorheilungsraten führten [3, 4]. Verlässliche Daten über die Langzeitmorbidität nach unkonventioneller Fraktionierung fehlen jedoch so dass derzeit keine abschließende Wertung möglich ist.

Ein Meilenstein in der experimentellen wie klinischen Strahlenbiologie war die Einführung des linear-quadratischen $\mathrm{Ge}$ setzes zur Beschreibung des zellulären Überlebens nach Be- 
Abb. 2. Prinzip der IMRT mittels «Step and shoot»-Technik. Subfelder werden unterschiedlich stark bestrahlt. Beispiel mit 6 Teilfeldern; oft wird mit 10-15 Teilfeldern gearbeitet. Bei konstanter Anordnung des Feldmittelpunkts ergibt sich eine (fast) beliebig zu gestaltenden Dosisverteilung im Summenfeld. Im Strahlenfeld liegende Gewebe hoher Strahlenempfindlichkeit wie z.B. das Rückenmark können so geschont werden.
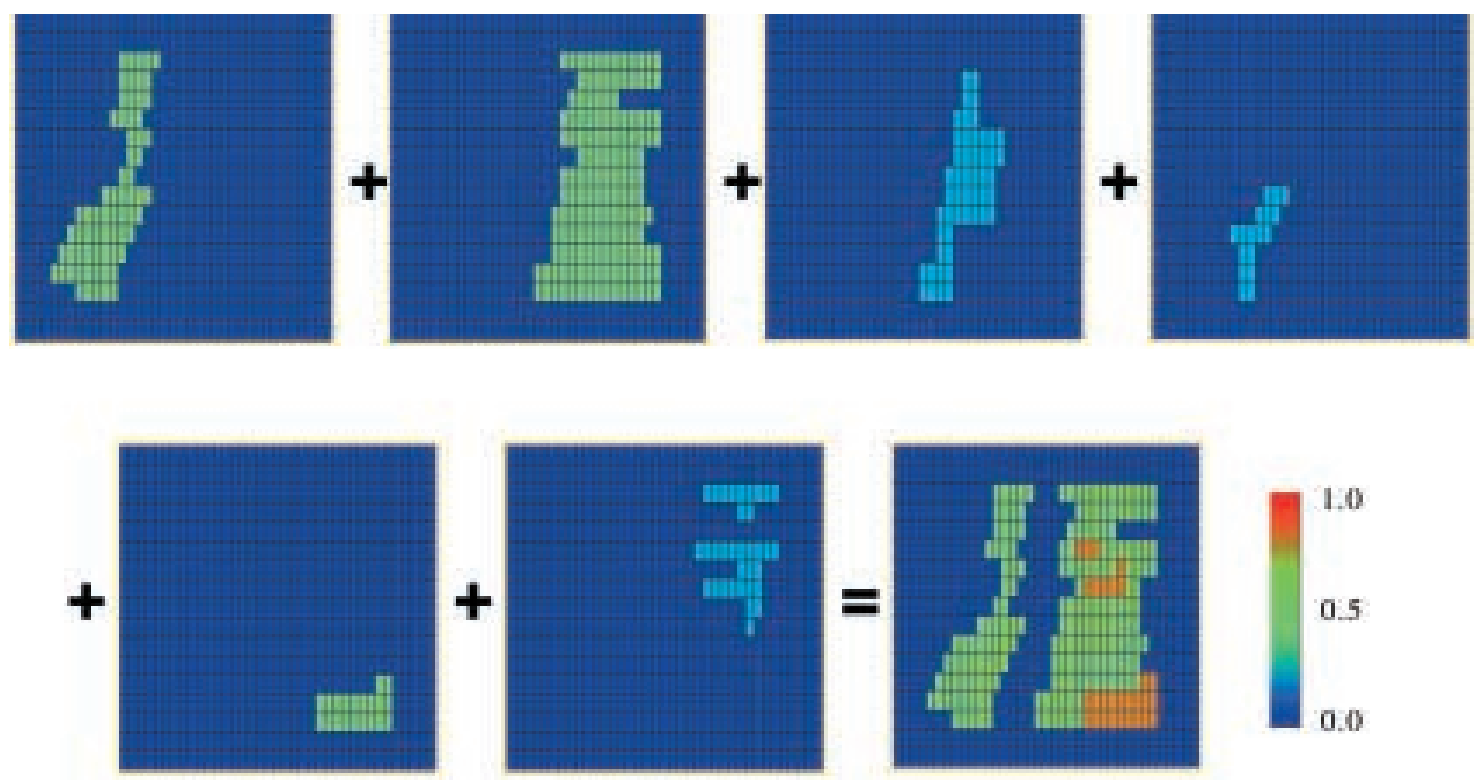

strahlung von Tumor- und Normalgeweben. Mit den 4 R's (Redistribution der Zellen innerhalb des Zellzyklus, Repopulierung, Reoxygenierung des Tumors, Reparatur des subletalen Strahlenschadens) wurde versucht, den Einfluss der Fraktionierung auf Tumor und Normalgewebe zu beschreiben [5]. Zusammen wurden daraus innovative Fraktionierungskonzepte wie die Bestrahlung mit drei Fraktionen pro Tag (CHART) abgeleitet [6], deren klinische Bedeutung derzeit in Studien geprüft wird und noch nicht abschließend beurteilt werden kann.

\section{Interaktion und klinischer Einsatz von ionisierenden Strahlen und dosismodifizierenden Substanzen (Zytostatika, Radiosensitizer)}

Als OnKOLOGIE erstmals erschien, war 5-Fluorouracil (5-FU) die wichtigste Substanz, die als Radiosensitizer eingesetzt wurde. Sie verdankte dies dem Umstand, dass nicht nur in vitro eine radiosensibilisierende Wirkung nachgewiesen werden konnte (wie bei vielen anderen Substanzen auch), sondern dass die simultane Radiochemotherapie bei Plattenepithelkarzinomen im Kopf-Hals-Bereich zu signifikant besseren 5- und 10-Jahres-Überlebensraten geführt hat [7]. Zur gleichen Zeit wurde erkannt, dass die Dauerinfusion über 4-5 Tage der Bolusapplikation überlegen war. Entsprechende Regime für verschiedene Tumoren sind bis heute aktuell. Unter den mannigfachen heute verfügbaren und in Kombination mit klinischer Strahlentherapie untersuchten Zytostatika nimmt Mitomycin C (MMC) insofern eine Sonderstellung ein, als dass kürzlich gezeigt werden konnte, dass MMC den negati- ven prognostischen Wert einer bestehenden Anämie bezügliche lokoregionärer Tumorkontrolle bei simultaner Radiochemotherapie ausgleicht [8].

\section{Integration der Radiotherapie in multimodale Therapie- protokolle}

Systematische posttherapeutische Nachbeobachtung und Analysen von Therapieergebnissen führten bei einer Vielzahl von Tumorenentitäten in bestimmten Stadien zu multimodalen Therapiekonzepten, die häufig die Strahlentherapie mit einschließen. Heute sind Radioonkologen fast immer sowohl in interdisziplinär besetzte fallbezogene Tumorkonferenzen wie auch in Studienkommissionen eingebunden.

Neuere Ergebnisse beim Rektumkarzinom haben gezeigt, dass gesteigerte chirurgische Radikalität (Exstirpation des Mesorektums) die (neoadjuvante) Radiotherapie zumindest in fortgeschrittenem Tumorstadium nicht immer ersetzen kann [9]. Von wenigen Ausnahmen abgesehen, hat sich die präoperative Radiotherapie jedoch nicht als generelles Therapieprinzip durchgesetzt. Obwohl randomisierte Studien fehlen, stellt die postoperative Radiotherapie lokoregionär fortgeschrittener Plattenepithelkarzinome im Kopf-Hals-Bereich eine allgemein akzeptierte Therapiemaßnahme dar. Insbesondere bei epithelialen Tumoren konnte durch die simultane Radiochemotherapie die lokoregionäre Rezidivrate bei einer Reihe von Hochrisikopatienten gesenkt und teilweise das tumorfreie Überleben verbessert werden. So kann die ungünstige Überlebensrate bei kapselüberschreitendem zervikalem Lymphknotenbefall, bei lokal fortgeschrittenen Tumoren, bei 


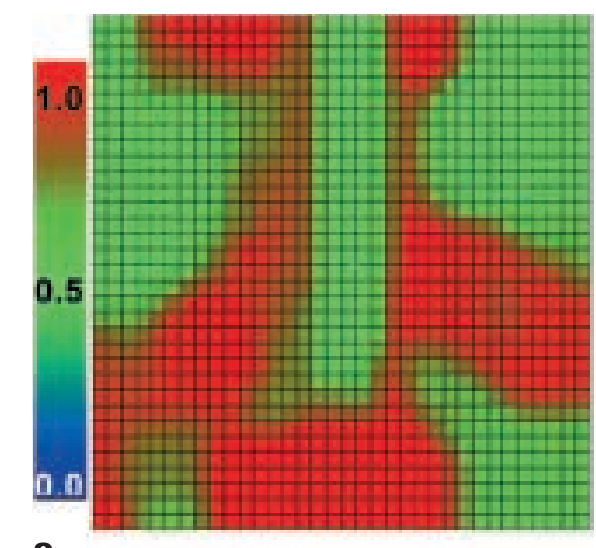

a

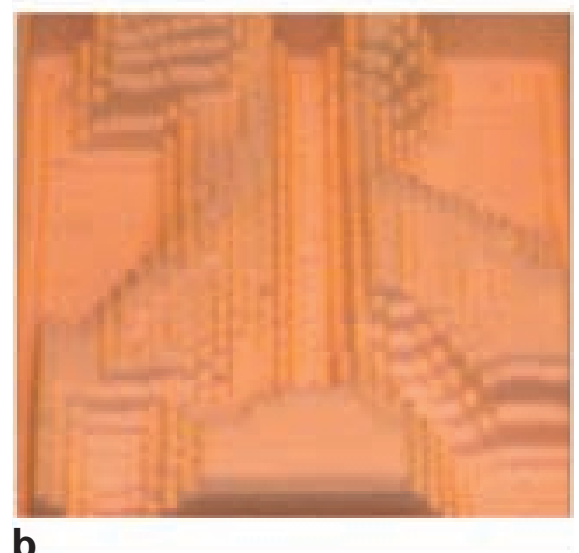

modulierte Radio-

therapie mit Kom-

pensatortechnik.

Entsprechend dem

berechneten Dosis-

fluenzprofils wird ein

3-dimensional ge-

formter Metallkom-

pensator hergestellt.

a Im Bestrahlungs-

planungsrechner be-

rechnetes Fluenzpro-

fil für ein Bestrah-

lungsfeld. b Styrodur-

Negativ für eine

Bestrahlungsfeld, was

mit Schwermetall

ausgegossen wird.

c Kontrolle der

Dosisverteilung am

Patienten mittels 15

MeV Röntgenstrah-

len (Therapiestrahl).

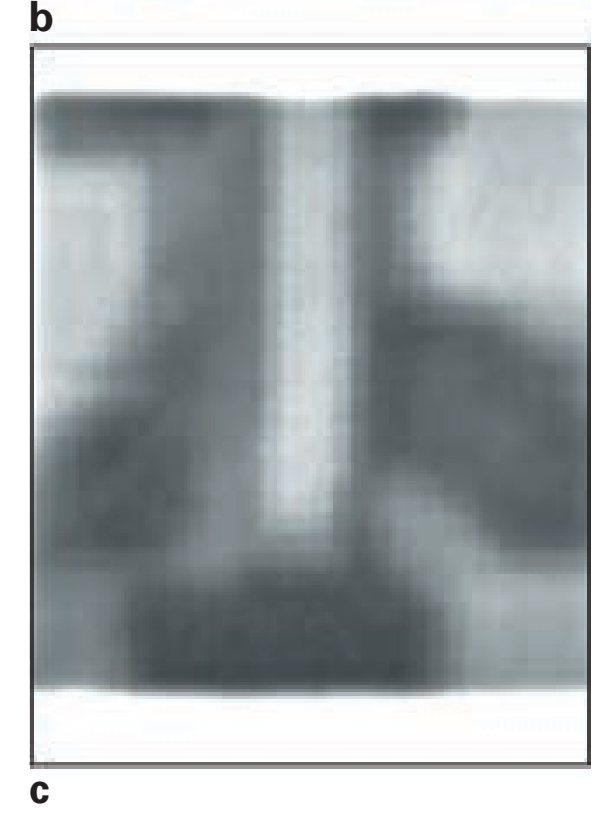

aus perkutaner und intrakavitärer Strahlentherapie mit simultaner Radiochemotherapie behandelt wurden. Seither hat sich diese Form der Behandlung als neuer Standard etabliert [13]. Bis in die 1960er Jahre hinein war die Diagnose Morbus Hodgkin auch bei Patienten, deren Erkrankung heute als frühes oder mittleres Stadium eingestuft werden würde, mit ungünstiger Langzeitprognose behaftet. Durch Einführung der Großfeldbestrahlung (Mantelfeld, inverses Y-Feld) unter Hochvoltbedingungen (Telekobalt-Strahlen) wurden aus Stanford, CA, Ende der 1960er Jahre deutlich bessere Heilungsraten berichtet als mit der bis dahin verbreiteten Einzelfeld bestrahlung. Als Band 1 dieser Zeitschrift erschien, war die «Extended-field»-Bestrahlung der erste Therapiestandard für M. Hodgkin in frühen Stadien. Zur Verminderung der Langzeittoxizität geheilter Patienten wurde nicht nur in den fortgeschrittenen, sondern auch in frühen Stadien eine Polychemotherapie der Bestrahlung vorgeschaltet, um damit Zielvolumen und Gesamtdosis zu vermindern. Damit konnte das krankheitsfreie Überleben (aber nicht das Gesamtüberleben) verbessert werden (Zwischenauswertung der HD-7-Studie der Deutschen Hodgkin-Lymphom-Studiengruppe, DHSG [14]). Parallel dazu wurde bei M.-Hodgkin-Erkrankungen im Kindesalter systematisch die Radiotherapie hinsichtlich Zielvolumen und Dosis eingeschränkt sowie auf Patienten mit Risikofaktoren begrenzt. Dabei erwies sich eine risikoadaptierte Radiotherapie mit 20 Gy als ebenso wirksam wie höhere Dosen; jedoch geht der völlige Verzicht auf die Bestrahlung mit einer erhöhten Rezidivrate einher (HD-95-Protokoll).

Die Ganzkörperbestrahlung zur Konditionierung vor Knochenmarktransplantation wurde in Deutschland nach ermutigenden Ergebnissen in Seattle,WA, etwa zeitgleich mit dem Erscheinen dieser Zeitschrift eingeführt. Die 1980er Jahre standen im Zeichen der klinischen Forschung zur Verminderung der akuten und chronischen Toxizität, so dass heute insbesondere der Lungentoxizität keine wesentliche Bedeutung mehr zukommt.

\section{Funktions- und Organerhalt durch kurative \\ Strahlentherapie}

\section{Mammakarzinom}

Seit Ende der 1970er Jahre hat die brusterhaltende Chirurgie und Nachbestrahlung die Mastektomie bei Mammakarzinomen im Stadium T1 unabhängig vom axillären Lymphknotenstatus ersetzt. Bis heute ist es nicht gelungen, eine Subgruppe von Patientinnen zu definieren, bei der auf die Bestrahlung der Restbrust verzichtet werden kann, ohne die Resultate zu verschlechtern.

\section{Kehlkopfkarzinom}

Die chirurgische Therapie von fortgeschrittenen Plattenepithelkarzinomen des Larynx aber auch des Hypopharynx
Patientinnen mit Tumoren des Gebärmutterhalses in den FIGO-Stadien II, III und IVA anstatt mit einer Kombination großem Tumorvolumen und bei Nachweis ausgedehnter Tubessert werden [10-12].

Mitte der 1990er Jahre wurden mehrere Studien abgeschlossen, die ein verbessertes 5-Jahres-Überleben zeigten, wenn 
Abb. 4. Dosisverteilung bei einem malignen Meningeom an der Schädelbasis. Links: 3-D-konformierende Bestrahlung mit 5 Feldern, rechts: 3-D-konformierende Bestrahlung mit 5 Feldern, davon 3 mit 3-DKompensatoren. Dadurch wird eine Dosisverminderung um $15 \%$ am Innenohr (Pfeil) erzielt und das Risiko einer radiogenen Spätfolge vermindert. Farbige Linien: Isodosen (entsprechend Legende).

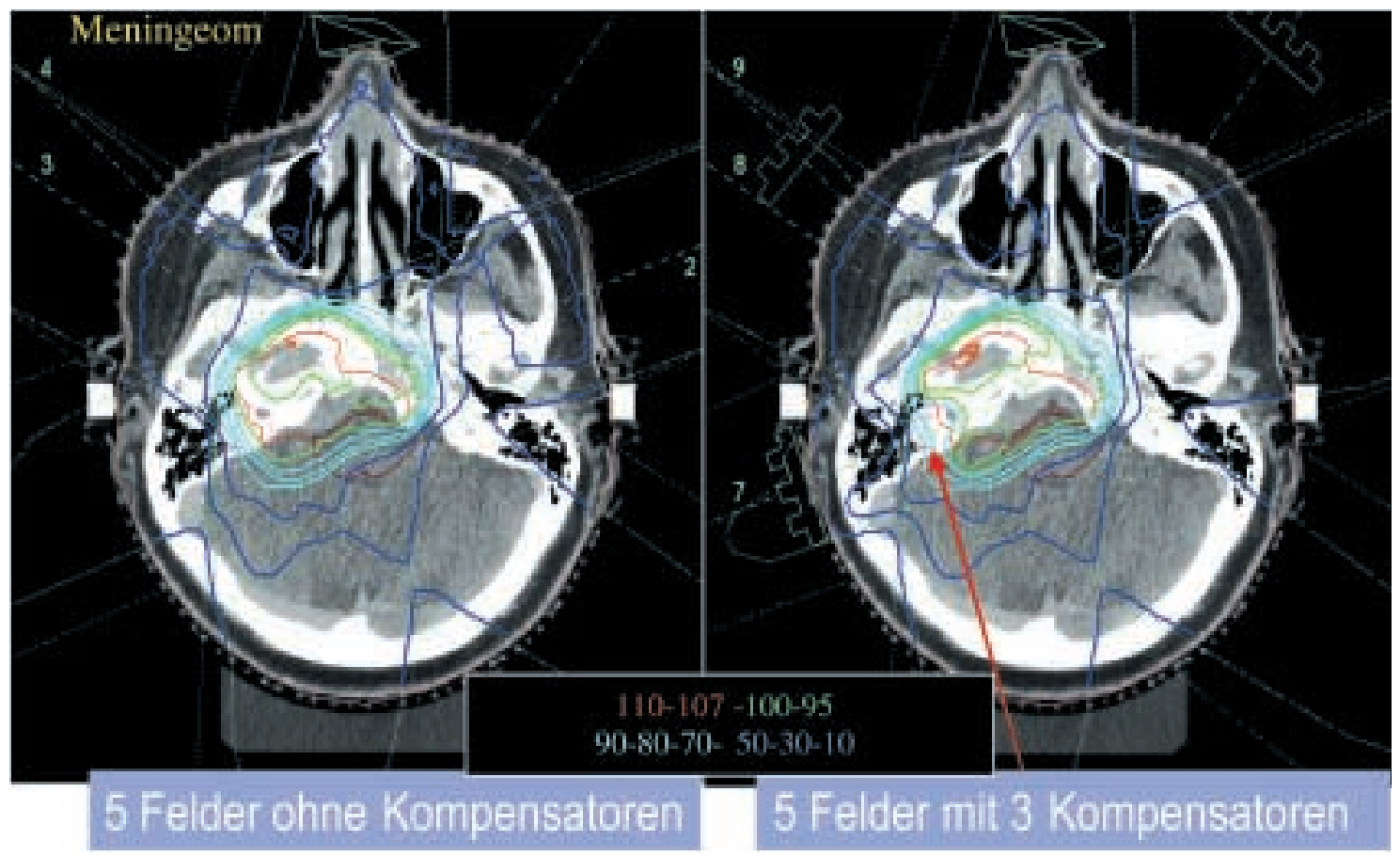

geht trotz Fortschritten der Laserchirurgie bei einem Teil der Patienten mit einer (mutilierenden) Laryngektomie einher. Mitte der 1990er Jahre konnte gezeigt werden, dass bei Patienten mit chemosensitiven Tumoren (Ansprechen auf eine Induktionschemotherapie, etwa $85 \%$ ) mit einer nachfolgenden Strahlentherapie eine 5-Jahres-Überlebensrate von knapp $50 \%$ erzielt werden kann, die mit der nach Laryngektomie vergleichbar ist. Bei diesem Konzept bleibt die Laryngektomie für die Rezidivtherapie reserviert. Im Gegensatz zur primären Laryngektomie kann mit dem konservativen Vorgehen bei $66 \%$ der Patienten der Kehlkopf erhalten werden [15].

\section{Analkarzinom}

Die Behandlungsergebnisse von Papillon und Montbarbon aus Lyon [16] läuteten einen Paradigmenwechsel in der Therapie des Plattenepithelkarzinoms des Analkanals ein. Mit Radiotherapie wurden der radikalen Chirurgie ebenbürtige 5-Jahres-Heilungsraten bei gleichzeitig hoher Rate an Kontinenzerhalt erzielt [16]. 20 Jahre nachdem der Chirurg Nigro über die Wirksamkeit der präoperativen simultanen Radiochemotherapie berichtete, erwies sich MMC als wesentlicher Bestandteil der heute als Standard anerkannten primären Radiochemotherapie [17], wobei die abdominosakrale Exstirpation für Rezidiv oder Tumorpersistenz reserviert bleibt.

\section{Prostatakarzinom}

Das Prostatakarzinom hat infolge des Aufkommens des prostataspezifischen Antikörpers im Serum als Tumormarker an Bedeutung zugenommen. In den 1980er und 1990er Jahren wurde die primäre, radikale, kurativ intendierte Strahlentherapie unter Einschluss der IMRT systematisch optimiert, so dass heute nach intensitätsmodulierter Radiotherapie mit Gesamtdosen von 70 Gy und höher geringe Langzeittoxizität und hohe Tumorkontrollraten (PSA im Nullbereich) erzielt werden können. Damit steht insbesondere für ältere Patienten (über 70 Jahre) eine therapeutische Option neben der radikalen Prostatektomie, die insbesondere für jüngere Patienten geeignet ist, zur Verfügung.

Die in der zurückliegenden Periode eingeleiteten technologischen Innovationen stehen, insbesondere was die IMRT anbelangt, an einem Anfang und besitzen mit der fortschreitenden Entwicklung von Hard- und Software großes Potenzial, die Strahlentherapie der Zukunft effektiver, aber auch nebenwirkungsärmer zu machen. Obwohl vereinzelt Daten auf einen systemischen Effekt der Strahlentherapie hinweisen [18], wird sie trotzdem vorrangig eine lokoregionäre Therapie für lokal begrenzte Krebsformen bleiben. Die biologischen Wirkungen der ionisierenden Strahlung auf Tumorgewebe ergänzen sich mit den Wirkungsmechanismen innovativer medikamentöser Therapiestrategien [19]. 


\section{Literatur}

1 Ling CC, Humm J, Larson S, Amols H, Fuks Z, Leibel S, Koutcher JA: Towards multidimensional radiotherapy (MD-CRT): Biological imaging and biological conformality. Int J Radiat Oncol Biol Phys 2000;47:551-560.

2 Salz H, Wiezorek T, Scheithauer M, Kleen W, Schwedas M, Wendt TG: Intensitätsmodulierte Strahlentherapie (IMRT) mit Kompensatoren. Med Phys 2002;(in press)

3 Fu KK, Pajak TF, Trotti A, Jones CU, Spencer SA, Phillips TL, Garden AS, Ridge JA, Cooper JS, Ang KK, for the RTOG A Radiation Therapy Oncology Group (RTOG): Phase III randomized study to compare hyperfractionation and two variants of accelerated fractionation to standard fractionation radiotherapy for head and neck squamous cell carcinomas: First report of RTOG 90003. Int J Radiat Oncol Biol Phys 2000;48:7-16.

4 Horiot JC, Le Fur R, N'Guyen T, Chenal C, Schrabu S, Alfonsi S, Gardiani G, Van den Bogaert W, Danczak S, Bolla M. Van Glabbeke M, De Pauw M: Hyperfactionation versus conventional fractionation in oropharyngeal carcinoma: Final analysis of a randomized trial of the EORTC cooperative group of radiotherapy. Radiother Oncol 1992;25: 231-241.

5 Withers HR: Biological basis for modifying conventional fractionation regimen in radiotherapy. Strahlenther 1984;160:670-677.

6 Dische S, Saunders M, Barrett A, Harvey A, Gibson D, Parmar M: A randomised multicentre trial of CHART versus conventional radiotherapy in head and neck cancer. Radiother Oncol 1997;44: 123-136.

7 Lo TC, Wiley AL Jr, Ansfield FJ, Brandenburg JH, Davis HL Jr, Gollin FF, Johnson RO, Ramirez G, Vermund H: Combined radiation therapy and 5fluorouracil for advanced squamous cell carcinoma of the oral cavity and oropharynx: A randomized study. Am J Roentgenol 1976;126:229-235.

8 Budach VG, Geismar D, Haake K, Stuschke M, Baumann M, Budach W, Grabenbauer GG, Wust P, Hinkelbein W, Wernecke KD: Hemoglobin is an independent prognostic factor in locally advanced head \& neck cancer - three year results from a German multicentre trial (ARO 95/6). Int J Radiat Oncol Biol Phys 2001;51(suppl):183, abstract no. 1014.

9 Kapiteijn E, Marijnen CA, Nagtegaal ID, Putter H, Steup WH, Wiggers, T, Rutten HJ, Pahlman L, Glimelius B, van Krieken JH, Leer JW, van de Velde CJ: Preoperative radiotherapy combined with total mesorectal excision for rectal cancer. N Engl J Med. 200;345:638-646.
10 Bernier J, Domenge C, Eschwege F, Ozsahin M, Matuszewska K, Moncho V, Greiner RH, Giralt J, Kirkpatrik A, van Glabbeke M: Chemo-radiotherapy, as compared to radiotherapy alone, significantly increases disease-free and overall survival in head and neck cancer patients after surgery: Results of EORTC Phase III Trial 22931. Int J Radiat Onco Biol Phys 2001;51(suppl):1, abstract no. plenary 1.

11 Grabenbauer GG, Steininger H, Meyer M, Fietkau R, Brunner T, Heinkelmann P, Hornung J, Iro H Spither W, Kirchener T, Sauer R, Distel L: Nodal CT density and total tumor volume as prognostic factors after radiation therapy of stage II/IV head and neck cancer. Radiother Oncol 1998;47:175-183.

12 Wendt TG: What is known in radiochemotherapy in advanced head and neck cancer? Strahlenther Onkol 1996;172:409-416.

13 Dunst J, Haensen G: Simultaneous radiochemotherapy in cervical cancer: Recommendations for chemotherapy. Strahlenther Onkol 2001;177:635640

14 Ansén S, Engert A, Wolf J, Sieber M, Paulus U, Diehl V: Deutsche. Hodgkin-Lymphom-Studiengruppe (DHSG): 20 Jahre Diagnostik, Therapie und Nachsorge von Patienten mit Morbus Hodgkin. Onkologie 2001;24(suppl 1):35-48.

15 Wolf GT, Hong WK, Fisher SG: Neoadjuvant chemotherapy for organ preservation: Current status. Proceedings of the 4th International Conference on Head and Neck Cancer 1996;4:89-97.

16 Papillon J, Montbarbon MD: Epidermoid carcinoma of the anal canal. Dis Colon Rectum 1987;30:324-333.

17 Flam MS, John M, Pajak TF, Petrelli N, Myerson R, Doggett S, Quivey J, Rotman M, Kerman H, Coia L, Murray K: Role of mitomycin in combination with fluorouracil and radiotherapy, and of salvage chemoradiation in the definitive nonsurgical treatment of epidermoid carcinoma of the anal canal Results of a phase III randomized Intergroup study. J Clin Oncol 1996;14:2527-2539.

18 Overgaard M, Hansen PS, Overgaard J, Rose C, Andersson M, Bach F, Kjaer M, Gadeberg CC, Mouridsen HT, Jensen MB, Zedeler K: Postoperative radiotherapy in high-risk premenopausal women with breast cancer who receive adjuvant chemotherapy. Danish Breast Cancer Cooperative Group 82b Trial. N Engl J Med 1997;337:949-955.

19 Robert F, Ezekiel MP, Spencer SA, Meredith RF, Bonner JA, Khazaeli MB, Saleh MN, Carey D, LoBuglio AF, Wheeler RH, Cooper MR, Waksal HW Phase I study of anti-epidermal growth factor receptor antibody cetuximab in combination with radiation therapy in patients with advanced head and neck cancer. J Clin Oncol 2001;19:3234-3243. 